\title{
Does sorption of sulfuryl fluoride by wheat reduce its efficacy against adults and eggs of Rhyzopertha dominica?
}

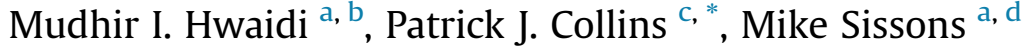 \\ a School of Environmental and Rural Science, The University of New England, Armidale, NSW 2351, Australia \\ ${ }^{\mathrm{b}}$ Department of Field Crops, College of Agriculture, University of Tikrit, Tikrit, Salahuddin, Iraq \\ c Department of Agriculture and Fisheries, Ecosciences Precinct, GPO Box 267, Brisbane, QLD 4001, Australia \\ ${ }^{\mathrm{d}}$ NSW Department of Primary Industries, Tamworth Agricultural Institute, 4 Marsden Park Road, Calala, NSW 2340, Australia
}

\section{A R T I C L E I N F O}

\section{Article history:}

Received 15 August 2017

Received in revised form

12 September 2017

Accepted 27 October 2017

\section{Keywords:}

Rhyzopertha dominica

Sorption

Sulfuryl fluoride

Wheat

\begin{abstract}
A B S T R A C T
Despite its growing importance as a fumigant for grain, there is no information on the impact of sorption on the efficacy of sulfuryl fluoride (SF) against target insect pests. Eggs and adults of a major grain pest, Rhyzopertha dominica, living in wheat (12\% m.c.), were fumigated with SF at $0.5,1$, and $2 \mathrm{mg} / \mathrm{L}$ for $168 \mathrm{~h}$ at $25{ }^{\circ} \mathrm{C}$. Sorption of the fumigant by the grain followed an exponential decay and reduced the mortality rates of both adults and eggs. The partition ratio $(K)$, a measure of physical sorption, had a strong impact on mortality of both adults and eggs. The quadratic model showed the best fit to the data and turning points in the relationship indicated that although mortality increased as concentration increased, physical sorption removed fumigant resulting in a decrease in the mortality rate. There was a linear relationship between mortality and the rate of sorption $(k)$ of SF by the wheat. At each concentration, mortality rate increased as $k$ increased despite sorption continuing, indicating that the chemical sorption rate had little impact on mortality. Sorption of SF into the commodity has the potential to reduce the biological efficacy of the fumigant resulting in potential control failures. Concentration $\mathrm{x}$ time protocols may need to be revised to account for this phenomenon.
\end{abstract}

Crown Copyright $\odot 2017$ Published by Elsevier Ltd. All rights reserved.

\section{Introduction}

Sulfuryl fluoride (SF) has been registered in the USA for more than 50 years and was originally used primarily as a treatment for pests of buildings, particularly termites (Osbrink et al., 1987). Its use expanded to replace methyl bromide in several applications when the latter began to be phased out of broad use under the Montreal Protocol Agreement (UNEP, 1994; Bell et al., 2003; Drinkall et al., 2004; Ducom, 2012). These applications included use in flour mills and food handling facilities, disinfestation of food commodities including cereal grains (Ducom, 2012), and dried fruit and nuts (Drinkall et al., 2004) in several countries.

Information on the efficacy of SF against insect pests is generally limited to fumigation times of $48 \mathrm{~h}$ or less, matching the usual application protocol for methyl bromide. Laboratory bioassays indicate that insect eggs are generally 5-30 fold more tolerant to SF

\footnotetext{
* Corresponding author.

E-mail addresses: mudhir76@yahoo.com (M.I. Hwaidi), pat.collins@daf.qld.gov. au (P.J. Collins), mike.sissons@dpi.nsw.gov.au (M. Sissons).
}

than other life stages. This has been demonstrated in beetle pests of museums (Su and Scheffrhan, 1990), wood boring beetles (Williams and Sprenkel, 1990), as well as beetle (Bell et al., 2003; Ciesla and Ducom, 2009; Jagadeesan and Nayak, 2016), moth (Baltaci et al., 2009; Bell and Savvidou, 1999) and psocid (Athanassiou et al., 2012) pests of stored products. Older eggs appear to be more tolerant than younger eggs (Baltaci et al., 2009; Williams and Sprenkel, 1990).

The lesser grain borer, Rhyzopertha dominica (Fab.), is a serious pest of stored wheat, barley and sorghum in Australia (Collins et al., 2017) and a major pest of stored cereals in warm temperate to tropical regions of the world (Edde, 2012). If left uncontrolled, it is capable of severely damaging or destroying grain stocks. In Australia and internationally, the fumigant phosphine is by far the preferred treatment for controlling infestations of $R$. dominica. However, serious levels of resistance in this pest to phosphine have been documented in several regions (Collins et al., 2017; Duong et al., 2016; Edde, 2012; Kaur et al., 2015; Opit et al., 2012) threatening the viability of phosphine. In Australia, SF has been adopted as an alternative treatment for control of phosphineresistant $R$. dominica and other pest species (Nayak et al., 2015). 
An important factor to consider in the practical application of fumigants is the impact of sorption of the gas into the commodity during fumigation. Sorption may reduce the biological activity of a fumigant by reducing the concentration of gas available to target insects (Banks, 1993). As demonstrated previously (Hwaidi et al., 2015), sorption of SF into commodities is significant, therefore, we reasoned that sorption may reduce the biological efficacy of this fumigant against target pests. The aim of our research was to determine the impact of sorption on the biological activity of SF against a major target pest, $R$. dominica.

\section{Materials and methods}

\subsection{Experimental design}

Eggs and adults of a phosphine resistant strain of $R$. dominica were fumigated with three different concentrations of SF: 0.5, 1.0 and $2.0 \mathrm{mg} / \mathrm{L}$ at exposure times ranging from 24 to $168 \mathrm{~h}$. The design was a randomized complete block with three replicates. Each replicate consisted of a glass Erlenmeyer flask (LabDirect, http://www.labdirect.com.au) of approximately 2.4 L capacity containing hard wheat grains ( $12 \%$ m.c.) filled to $50 \%$ flask volume (filling ratio of 0.50 ) and equilibrated for 2 days at $25^{\circ} \mathrm{C}$. Flasks were sealed with a glass stopper containing a silicone rubber septum to facilitate addition of fumigant and gas sampling.

\subsection{Insects and commodity}

$R$. dominica strain QRD569 was used for all experiments. This strain had been maintained in the laboratory since 1997 when it was collected from a phosphine fumigation control failure. QRD569 is classified as strongly resistant to phosphine (Collins et al., 2002) and was cultured on organic whole wheat at $30^{\circ} \mathrm{C}$ and $60 \% \mathrm{rh}$. The wheat, Triticum aestivum, (hard white, cultivar Gregory) used in these experiments was purchased from a certified organic producer on the Darling Downs, southeast Queensland, Australia and stored in $20 \mathrm{~kg}$ bags at $-20{ }^{\circ} \mathrm{C}$. The wheat was brought to laboratory temperature and allowed to equilibrate for 2 days before conditioning to $25{ }^{\circ} \mathrm{C}$ and $12 \%$ moisture content (AACC, 2010).

\subsection{Fumigations}

Fumigations were undertaken as described by Hwaidi et al. (2015). Briefly, SF (99.8\% purity) was decanted from a 3 L cylinder into a Flex foil gas-sampling bag (SKC, www.skcinc.com) from which various volumes of SF could be withdrawn using gas-tight syringes (Hamilton, Grace Discovery Sciences, Epping, VIC., Australia) for bioassay. SF concentration was measured using a gas chromatograph (Perkin Elmer, Claris 500) fitted with a thermal conductivity detector (TCD).

Glass Erlenmeyer flasks of known volume (about 2.4 L) were filled to $50 \%$ volume with wheat of $12 \%$ m.c., and 100 adult $R$. dominica (a mixture of males and females, 2-4 weeks posteclosion) were placed in each flask. The insects were left for 4 days prior to fumigation to allow the adults time to lay eggs. The desired concentration of SF was added to each flask using a gastight syringe. The flasks were placed in a controlled environment room for up to $168 \mathrm{~h}$ at $25{ }^{\circ} \mathrm{C}$ and $60 \% \mathrm{rh}$. Gas samples $(5 \mu \mathrm{L})$ were removed from the headspace of each experimental flask using a gas tight syringe at $24 \mathrm{~h}$ intervals. For each replicate, three samples were taken at each time interval and the average used as the reading. As the experimental SF concentrations were much lower than the range measured by a TCD, a more sensitive flame photometric detector was used (Hwaidi et al., 2015).

After completion of the fumigation, flasks were removed from the controlled environment room, opened and aerated under a fume hood for $2 \mathrm{~h}$ to disperse the gas. The wheat and insects were gently sieved using a $2 \mathrm{~mm}$ mesh sieve to separate all adults from the grain. The grain was carefully inspected to ensure that all adults had been removed. After making sure that 100 adults were retrieved, the adults were allowed a 7-day recovery period then scored for mortality and destroyed.

The grain, which now contained eggs laid by the adults before the fumigation, was transferred to 2 L plastic jars (Atlas Plastics, Minto NSW, Australia). A hole $(50 \mathrm{~mm})$ was drilled into the lid of each plastic jar and a white filter paper circle $(110 \mathrm{~mm}$, Advantec, Toyo Roshi Kaisha, Japan) and a piece of gauze were placed across the neck of the jar before screwing on the lid, to facilitate gaseous exchange. The jars were stored at $25^{\circ} \mathrm{C}$ and $60 \%$ relative humidity for 8 weeks to allow all eggs sufficient time to develop into adults. After the 8 weeks, the $2 \mathrm{~L}$ plastic jars were opened to count the number of adults remaining.

\subsection{Controls}

\subsubsection{Fumigant}

A set of control flasks, containing SF in air only, were monitored during the experiment to detect possible loss of fumigant. There were 9 flasks, three for each test concentration. The control flasks were also used as the reference for creating calibration curves before measuring sulfuryl fluoride concentration in the headspace of bioassay flasks. To facilitate this, one of the three flasks had the desired test concentration and the other two had a concentration either higher or lower than the test concentration. For example, for $2 \mathrm{mg} / \mathrm{L} \mathrm{SF}$, there was one flask with $1.8 \mathrm{mg} / \mathrm{L}$, a second containing, $2.0 \mathrm{mg} / \mathrm{L}$ and a third containing $2.2 \mathrm{mg} / \mathrm{L}$.

\subsubsection{Insects}

Control flasks consisted of wheat and adult insects subjected to the same conditions as the SF treated flasks but not fumigated. There were three flasks (replicates) for each of the eight time points during the study.

\subsection{Calculating initial fumigant concentration and commodity volume}

As described previously (Hwaidi et al., 2015) the initial concentration of SF at zero time $\left(C_{0}\right)$ was calculated by multiplying the volume to be added by the source concentration divided by the total space (after adding the commodity):

$C_{0}=C_{f}^{*} C_{s} / V$

where

$C_{f}=C_{d}{ }^{*} V / C_{s}$

$C_{d}=$ desired concentration. $V=$ the volume of the fumigation flask. $C_{s}=$ gas concentration of the source. $C_{d}=C_{0}$, in this case.

The space remaining in each flask after adding the commodity was calculated by dividing the bulk density $\left(\rho_{\text {bulk }}\right)$ by the true density $\left(\rho_{\text {true }}\right)$.

$S=1-\rho_{\text {bulk }} / \rho_{\text {true }}$

\subsection{Statistical analysis}

Data were analysed using SAS software (SAS, 1999). The decay of 
gas concentrations and egg and adult numbers were fitted to mathematical models described below:

The decrease in gas concentration was best described as the exponential relationship:

$C_{t}=C_{0} e^{-k^{*} t}$

where $C_{t}$ is the gas concentration $C$ at time $t . C_{0}$ is the gas concentration $C$ at time zero $\left(t=t_{0}=0\right)$. At $t=0$ the sorption rate constant $k=0$ because when substituting zero into equation number (4) and multiplying the zero by $k$, gives zero sorption. In this case $C_{t}=C_{0}$.

Adult (parent) mortality was described as:

$M_{t}=M_{0} e^{-R^{*} t}$

Where $M_{t}$ was the number of adults $M$ at any time $t$ and $R$ is the rate constant for adult mortality. $M_{0}$ was adult number $M$ at time zero $\left(t=t_{0}=0\right)$.

Egg survival was described as:

$N_{t}=N_{0} e^{-E^{*} t}$

where $\left(N_{t}\right)$ is the number of adult progeny $(N)$ at a given time $(t)$. $\left(N_{0}\right)$ is the number of progeny at time zero $\left(t=t_{0}=0\right)$. $E$ is the rate constant of adult progeny hatched from fumigated eggs. When substituting $(t)$ for zero in equation (6) and multiplying by $(E)$, the result would be zero. In this case $N_{t}=N_{0}$.

The sorption rate for a full container, $k_{f}$, and the tendency to take up fumigant by physical sorption, known as the partition ratio (or co-efficient) of physical sorption, $K$, were each calculated following the methods of Hilton and Banks (1997). The partition ratio gives a dimensionless measure of the tendency of the commodity to take up fumigant by physical sorption (Hilton and Banks, 1997).

$k_{f}=k / f$

where $k$ was sorption rate constant from equation (4), and $f$ was the experimental filling ratio.

$K=\left(C_{a}-C_{i}\right)^{*} V_{g} / C_{i}^{*} V_{f}$

where $C_{a}$ was the applied concentration, $C_{i}$ was the predicted concentration, $V_{g}$ was the gas volume and $V_{f}$ was the volume occupied by the commodity.

The relationships between the partition ratio, $K$, and the egg survival rate constant, $E$, and the adult mortality rate constant, $R$, were fitted using a polynomial quadratic regression.

$E=B_{0}+B_{1} K+B_{2} K^{2}$

$R=B_{0}+B_{1} K+B_{2} K^{2}$

Where $B_{0}$ was the intercept, $B_{1}$ was the linear coefficient and $B_{2}$ was the quadratic coefficient.

The turning point of the standard polynomial quadratic regression line was calculated using the following equation.

$K=-B_{1} / 2 B_{2}$

The turning point of the fitted line is the point at which the line turns back toward zero. Therefore, it is considered to be the point of maximum partition of physical sorption $(K)$. This gives the maximum egg survival $(E)$ or adult mortality $(R)$ rate constant. After calculating $K$, equations (9) and (10) were used to calculate the maximum rate of mortality by substituting the derived values of $K$ into each equation.

A linear regression formula was used to describe the relationship between both the egg survival rate $(E)$ and the adult mortality rate $(R)$ with the sorption rate $(k)$.

$E=B_{0}+B_{1}(k)$

$R=B_{0}+B_{1}(k)$

In addition, the same linear equation was used to describe the relationship between egg survival $\left(E_{g}\right)$ and exposure time $(t)$ when the fumigant was not present.

$E_{g}=B_{0}+B_{1}(t)$

The percentage of gas loss, egg survival and adult mortality per $h$ were calculated using the following equation:

Percentage $=100\left(1-\mathrm{e}^{i}\right)$

where $(i)$ was $(k, E$ or $R)$.

Finally, time to 50 (equation (9)) and 99\% (equation (10)) loss of the gas, and egg survival and adult mortality were calculated according to the following:

$1 / 2 L_{0}=L_{0} e^{-i^{*} t} / L_{0}, 1 / 2=e^{-i^{*} t}, t=\ln (1 / 2) /-i$.

$0.01 L_{0}=L_{0} e^{-i^{*} t} / L_{0}, 0.01=e^{-i^{*} t}, t=\ln (0.01) /-i$.

where, $L_{0}$ was $C_{0}, N_{0}$ or $M_{0}, i$ was $k, E$ or $R$ and ln was the natural logarithm.

\section{Results}

\subsection{Sorption}

SF concentration was very stable in the control flasks over the period of the study, indicating negligible leakage and providing confidence in the calibration curves used for measuring SF at each time interval. The mean readings and standard deviations for SF concentrations at $0.5,1.0$ and $2.0 \mathrm{mg} / \mathrm{L}$ across all time intervals were $0.48 \pm 0.02,0.98 \pm 0.017$ and $1.94 \pm 0.21$, respectively.

The concentration of SF in the headspace of fumigated flasks containing wheat with $R$. dominica adults and eggs present declined with time in each treatment (Fig. 1). This decline indicates that the fumigant was sorbed into the wheat, otherwise the concentration would have remained stable, as occurred in the controls. Sorption was initially rapid followed by a slowing of the rate. The rate of decline differed between the three SF concentrations and were compared by fitting an exponential model, which provided a highly significant fit to the data with all treatments (Table 1). The decline in SF concentration over time was fastest at 0.5 and slowest at $2.0 \mathrm{mg} / \mathrm{L}$. The calculated (from equations (9) and (10)) time to 50 and $99 \%$ loss of SF increased with increasing applied SF concentration (Table 1).

\subsection{Adult mortality}

Fumigating with SF resulted in an exponential decline in numbers of adult $R$. dominica over time (Fig. 2). The mortality rate constant for adults $(R)$ increased with increasing SF concentration. However, complete control of adults was not achieved in the presence of wheat even at the highest concentration $(2 \mathrm{mg} / \mathrm{L})$ of SF tested (Fig. 2). The exposure period required at $2 \mathrm{mg} / \mathrm{L}$ for complete 


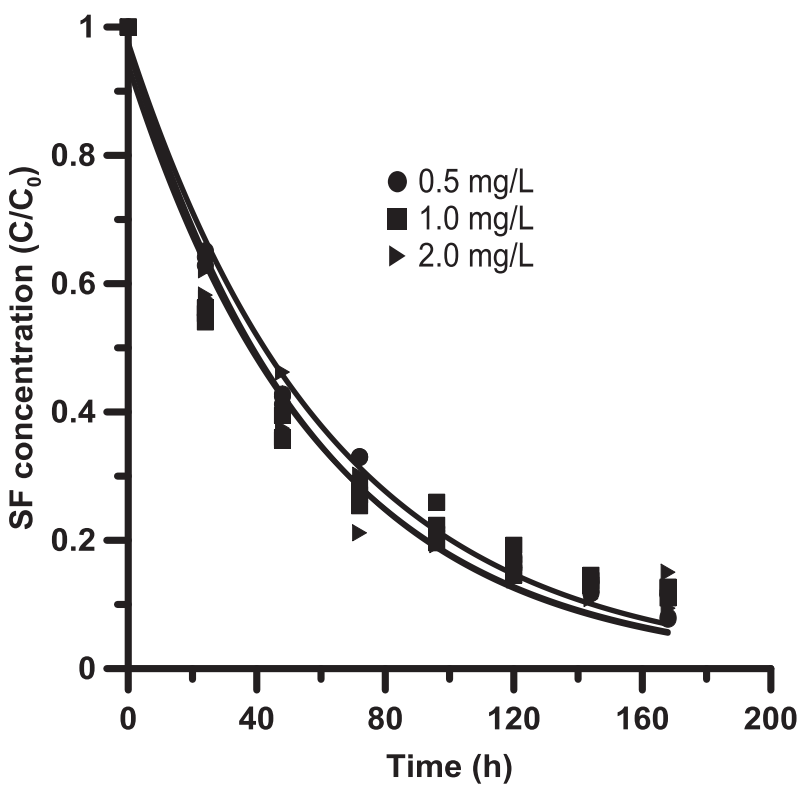

Fig. 1. Sulfuryl fluoride concentration in the head space of flasks containing wheat $(12 \% \mathrm{mc})$ and Rhyzopertha dominica adults and eggs fumigated with $0.5,1.0$ or $2.0 \mathrm{mg} / \mathrm{L}$ at $25{ }^{\circ} \mathrm{C}$ and $60 \%$ rh for $168 \mathrm{~h}$.

control can be predicted from the regression equation (Table 2). Thus, the exposure time would need to be extended to $422 \mathrm{~h}$ to achieve $100 \%$ mortality of adults with $2 \mathrm{mg} / \mathrm{L} \mathrm{SF}$, while $99 \%$ of the fumigant would be lost after $820 \mathrm{~h}$ (Table 1 ).

The partition ratio $(K)$ had a strong impact on the adult mortality rate, $R$, and this effect was stronger at the lower SF concentrations, that is, $K$ and $R$ were larger in magnitude at 0.5 than at $2 \mathrm{mg} / \mathrm{L}$ (Fig. 3). The quadratic model provided the best fit for this relationship with high $R^{2}$ values of $0.94-0.97$ (Table 3 ). The relationship between $K$ and $R$ showed turning points at which the rate of adult mortality $R$ began to decrease as $K$ values increased (Fig. 3, Table 3).

Adult survival rate $(R)$ increased as sorption rate $(k)$ increased in absolute value. A linear model, with excellent fit of the data (Table 4), best described the relationship between $R$ and $k$. At each concentration, $R$ continued to increase as $k$ increased despite sorption continuing, indicating that the chemical sorption rate had little impact on adult mortality and that the greatest impact was due to loss of fumigant through physical sorption. For example, increasing $k$ by one unit leads to an increase in $R$ by $0.142,0.731$ and 1.930 at $0.5,1$ and $2 \mathrm{mg} / \mathrm{L}$, respectively (Table 4).

\subsection{Egg survival}

Number of adult progeny and therefore egg survival decreased with increasing concentrations and as fumigation time increased at each concentration. Egg survival occurred in all treatments, even at the highest concentration tested, $2 \mathrm{mg} / \mathrm{L}$ for $168 \mathrm{~h}$

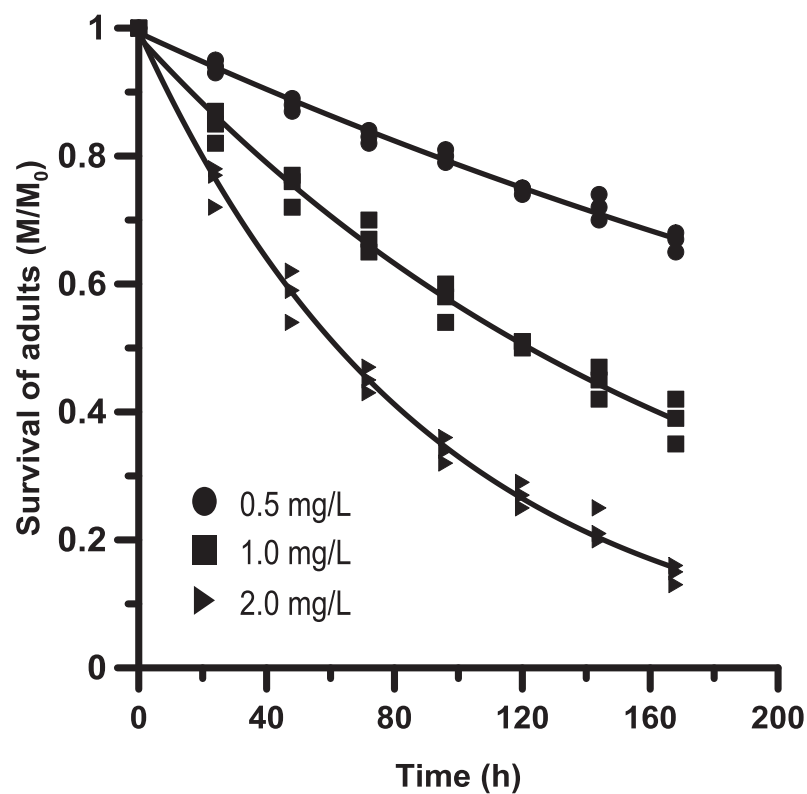

Fig. 2. Survival of adults of Rhyzopertha dominica relative to controls in wheat (12\% mc) fumigated with $0.5,1$ or $2 \mathrm{mg} / \mathrm{L}$ sulfuryl fluoride at $25^{\circ} \mathrm{C}$ and $60 \% \mathrm{rh}$.

(Table 2). The rate constant of egg mortality $(E)$ closely fitted the exponential model, explaining 98-99\% variation in the data (Table 2). Egg mortality was strongly affected by sorption of the fumigant into the commodity. However, in the control, the numbers of adult progeny emerging from the grain (non-fumigated treatment) increased rapidly with time (Fig. 4). In contrast to the situation with adults, these curves predict that, at $2 \mathrm{mg} / \mathrm{L}$, $99 \%$ of the fumigant would be lost before $99 \%$ control of eggs could be achieved.

The relationship between egg mortality rate $(E)$ and the partition ratio of physical sorption $(K)$ was curvilinear at the three concentrations tested. This relationship was best explained by the quadratic model, which was an excellent fit for the data $\left(R^{2}=0.95-0.99\right)$ (Table 3$)$. The turning points of the fitted lines (Table 3) revealed an increase of $E$ values with increasing applied SF concentration, but showed a decrease in $K$ values. Thus, although egg mortality rate initially increased as concentration increased, physical sorption removed fumigant resulting in a decrease in the rate of mortality until it reached zero, and then the numbers of eggs surviving began to increase. As expected, maximum or turning point $K$ values obtained for adult and eggs were identical as they are derived from the same fumigation (Table 3 ). However, $E$ values were lower than $R$ values reflecting the higher sensitivity of adults to the fumigant (Table 3 ).

The relationship between sorption rate $(k)$ and egg mortality rate $(E)$ was linear at each concentration tested (Fig. 5, Table 4). For example, when SF was $0.5 \mathrm{mg} / \mathrm{L}$, mortality increased to about 0.061 with an increase in $k$ of one unit, and when SF was $2 \mathrm{mg} / \mathrm{L}$, mortality increased by about 0.76 with each increase in $k$ of one unit.

Table 1

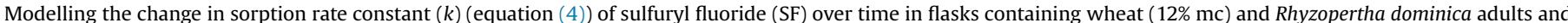

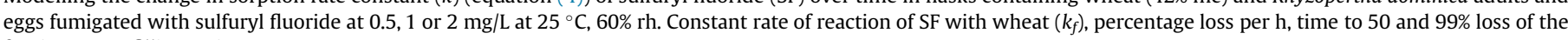
fumigant at a filling ratio 0.5 .

\begin{tabular}{|c|c|c|c|c|c|c|c|}
\hline $\mathrm{SF} \mathrm{mg} / \mathrm{L}$ & Regression & Significance & $R^{2}$ & $\%$ loss SF per $h$ & Time (h) to $50 \%$ loss of SF & Time (h) to $99 \%$ loss of SF & $k f$ \\
\hline 0.5 & $C_{t}=0.4547 e^{-0.016 t}$ & $F_{2,22}=366.91 ; P<0.0001$ & 0.92 & 1.610 & 43.04 & 286.03 & 0.0322 \\
\hline 1 & $C_{t}=0.9584 e^{-0.007 t}$ & $F_{2,22}=994.11 ; P<0.0001$ & 0.93 & 0.749 & 92.52 & 614.16 & 0.0149 \\
\hline 2 & $C_{t}=1.9823 e^{-0.005 t}$ & $F_{2,22}=1191.65 ; P<0.0001$ & 0.90 & 0.561 & 123.52 & 820.08 & 0.0112 \\
\hline
\end{tabular}


Table 2

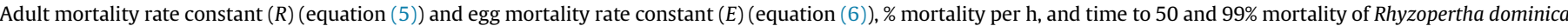
adults and eggs in wheat $(12 \% \mathrm{mc})$ fumigated with sulfuryl fluoride $(\mathrm{SF})$ at $0.5,1$ or $2 \mathrm{mg} / \mathrm{L}$ at $25{ }^{\circ} \mathrm{C}$ and $60 \%$ rh at filling ratio 0.5 .

\begin{tabular}{|c|c|c|c|c|c|c|}
\hline $\mathrm{SF} \mathrm{mg} / \mathrm{L}$ & Regression & Significance & $R^{2}$ & $\%$ Mortality per $\mathrm{h}$ & Time (h) to $50 \%$ mortality & Time (h) to $99 \%$ mortality \\
\hline \multicolumn{7}{|c|}{ Adult mortality } \\
\hline 0.5 & $M_{t}=98.87 e^{-0.002 t}$ & $F_{2,22}=595 ; P<0.0001$ & 0.96 & 0.229 & 301.30 & 2002.24 \\
\hline 1 & $M_{t}=98.19 e^{-0.005 t}$ & $F_{2,22}=109 ; P<0.0001$ & 0.93 & 0.550 & 125.54 & 834.26 \\
\hline 2 & $M_{t}=98.74 e^{-0.010 t}$ & $F_{2,22}=715 ; P<0.0001$ & 0.98 & 1.084 & 63.57 & 422.49 \\
\hline \multicolumn{7}{|c|}{ Egg mortality } \\
\hline 0.5 & $N_{t}=2.543 e^{-0.001 t}$ & $F_{2,19}=223.92 ; P<0.0001$ & 0.99 & 0.099 & 693.00 & 4605.17 \\
\hline 1 & $N_{t}=2.392 e^{-0.002 t}$ & $F_{2,19}=343.18 ; P<0.0001$ & 0.98 & 0.209 & 330.01 & 2192.93 \\
\hline 2 & $N_{t}=2.3475 e^{-0.004 t}$ & $F_{2,19}=151.20 ; P<0.0001$ & 0.99 & 0.439 & 157.50 & 1047.02 \\
\hline
\end{tabular}

The egg mortality rate constant $(E)$ was lower than the adult mortality rate $(R)$ at all concentrations tested, demonstrating that eggs were more tolerant than adults to SF (Table 2). Thus, the time required to control adults was much shorter than needed to control eggs. For example, at $2 \mathrm{mg} / \mathrm{L}, R$ was 0.01 per h, whereas $E$ was 0.004 per $h$. The adult mortality rate constant $(R)$ increased significantly with increasing fumigant concentration (Table 2) and time to 50 and $99 \%$ mortality decreased with increasing SF concentration. The increase in $E$ was much slower than the increase in $R$ with fumigant concentration (Table 2).

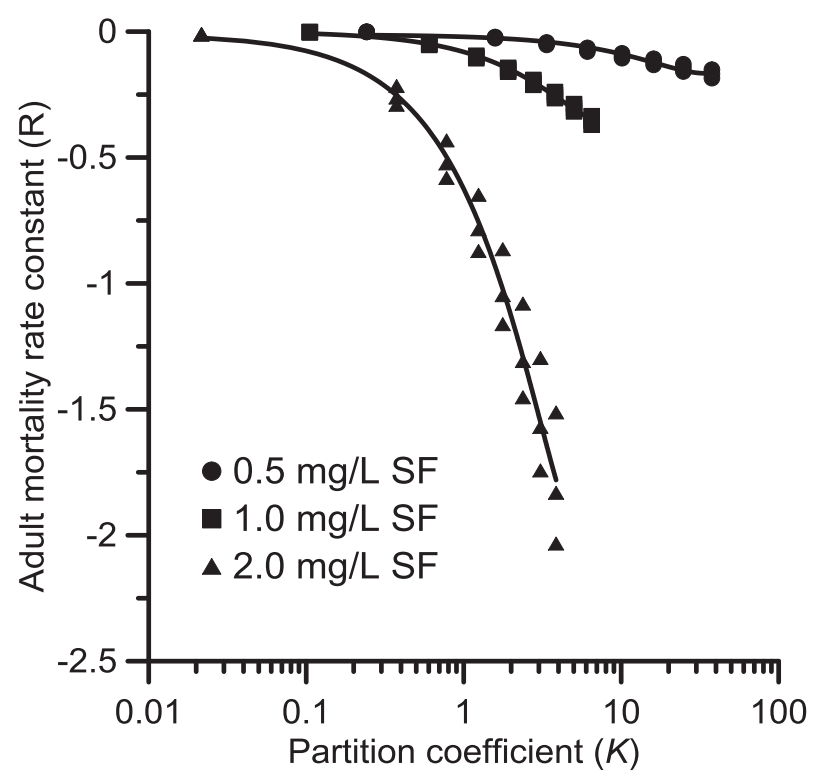

Fig. 3. Relationship between the partition coefficient for physical sorption $(K)$ of wheat $(12 \% \mathrm{mc})$ fumigated at three concentrations of sulfuryl fluoride (SF) and the adult mortality rate constant $(R)$ for Rhyzopertha dominica.

\section{Discussion}

The aim of this study was to test the hypothesis that sorption into the commodity during fumigation would reduce the efficacy of SF against $R$. dominica, a major pest of stored grain. Sorption was characterised using the partition ratio $(K)$ sorption rate constant $(k)$ of the chemical reaction between SF and grain. These factors were compared with the mortality rate constants for the egg $(E)$ and adult $(R)$ life stages.

The results of these experiments indicate that sorption into wheat led to a decrease in the efficacy of SF against eggs and adults of $R$. dominica. Wheat grains sorbed SF in an exponential manner and the data closely fitted first order exponential decay equations describing the relationship between fumigant sorption and exposure time as discussed in detail previously (Hwaidi et al., 2015). There was a rapid initial (first $24 \mathrm{~h}$ ) decline in headspace concentration of SF (Fig. 1), which is typical of fumigant sorption behaviour (Banks, 1993; Daglish and Pavic, 2008; Hilton and Banks, 1997). The sorption rate constant $(k)$ was independent of the applied

Table 4

Fit of the model for the relationship between the constant rate of reaction $(k)$ and the mortality rate constant for adults $(R)$ and eggs $(E)$ in wheat $(12 \%$ m.c., filling ratio $50 \%$ ) fumigated with sulfuryl fluoride (SF) at three different concentrations at $25{ }^{\circ} \mathrm{C}$ and $60 \%$ r.h. Eg is the model for population growth rate constant for insects in control (non-fumigated) wheat.

\begin{tabular}{clll}
\hline SF mg/L & Linear regression & Significance & $R^{2}$ \\
\hline \multicolumn{2}{c}{ Adult mortality } & & \\
0.5 & $R=(-0.00171)+(0.14278)(k)$ & $F_{1,22}=247 ; P<0.0001$ & 0.98 \\
1 & $R=(-0.00359)+(0.73187)(k)$ & $F_{1,22}=281 ; P<0.0001$ & 0.96 \\
2 & $R=(-0.00583)+(1.93060)(k)$ & $F_{1,22}=243 ; P<0.0001$ & 0.95 \\
Egg mortality & & \\
0.5 & $E=(-0.00191)+(0.06121)(k)$ & $F_{1,22}=360.64 ; P<0.0001$ & 0.94 \\
1 & $E=(-0.00247)+(0.27768)(k)$ & $F_{1,22}=852.77 ; P<0.0001$ & 0.97 \\
2 & $E=(-0.00180)+(0.76267)(k)$ & $F_{1,22}=4565 ; P<0.0001$ & 0.99 \\
Control & & & \\
0 & $E g=(0.011)+(2.307)(t)$ & $F_{1,22}=815.33 ; P<0.0001$ & 0.97 \\
\hline
\end{tabular}

Table 3

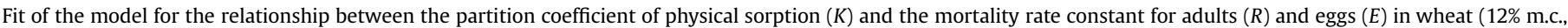

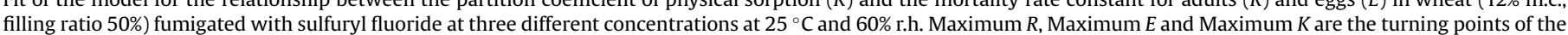
fitted equation.

\begin{tabular}{|c|c|c|c|c|c|c|}
\hline $\mathrm{SF} \mathrm{mg} / \mathrm{L}$ & Orthogonal polynomial regression & Significance & $R^{2}$ & Maximum $R$ & Maximum $E$ & Maximum $\mathrm{K}$ \\
\hline \multicolumn{7}{|c|}{ Adult mortality } \\
\hline 0.5 & $R=(-0.02692)+(-0.02084)^{*}(\mathrm{~K})+(0.00030670)^{*}(\mathrm{~K})^{2}$ & $F_{2,21}=499 ; P<0.0001$ & 0.97 & -0.381 & & 33.970 \\
\hline 1 & $R=(0.00359)+(-0.22799)^{*}(\mathrm{~K})+(0.01334)^{*}(\mathrm{~K})^{2}$ & $F_{2,21}=855 ; P<0.0001$ & 0.99 & -0.972 & & 8.545 \\
\hline 2 & $R=(-0.01067)+(-0.68295)^{*}(\mathrm{~K})+(0.05554)^{*}(\mathrm{~K})^{2}$ & $F_{2,21}=292 ; P<0.0001$ & 0.94 & -3.867 & & 6.148 \\
\hline \multicolumn{7}{|c|}{ Egg mortality } \\
\hline 0.5 & $E=(-0.01171)+(-0.00906)^{*}(\mathrm{~K})+(0.00013335)^{*}(\mathrm{~K})^{2}$ & $F_{2,21}=239.29 ; P<0.0001$ & 0.95 & & -0.167 & 33.971 \\
\hline 1 & $E=(0.00132)+(-0.08705)^{*}(\mathrm{~K})+(0.00509)^{*}(\mathrm{~K})^{2}$ & $F_{2,21}=1664 ; P<0.0001$ & 0.99 & & -0.371 & 8.551 \\
\hline 2 & $E=(-0.00421)+(-0.26942)^{*}(\mathrm{~K})+(0.022191)^{*}(\mathrm{~K})^{2}$ & $F_{2,21}=6688 ; P<0.0001$ & 0.99 & & -0.832 & 6.148 \\
\hline
\end{tabular}




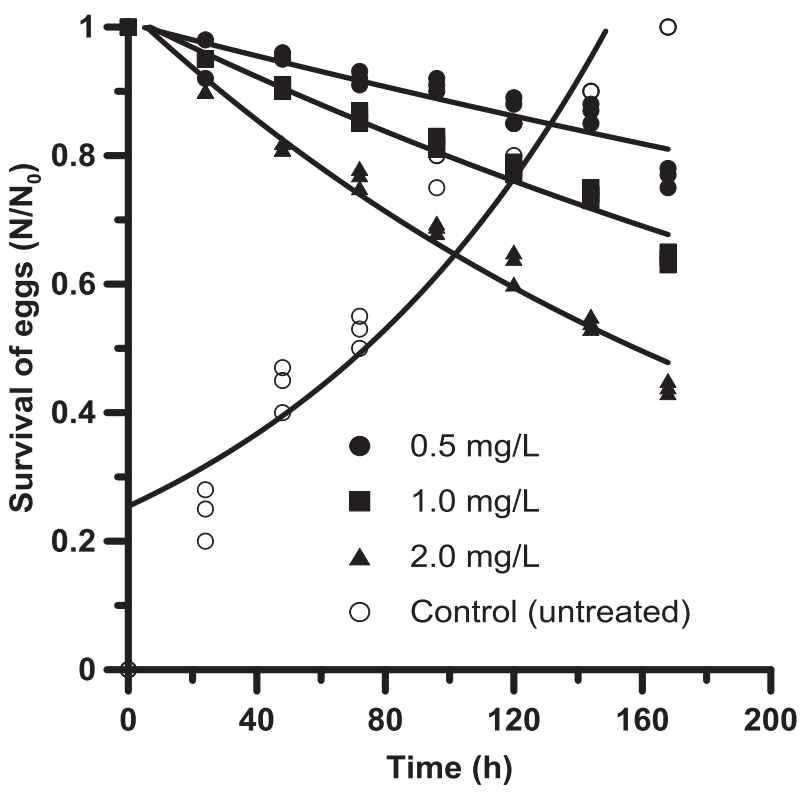

Fig. 4. Survival of eggs, measured as adult progeny, of Rhyzopertha dominica relative to controls in wheat $(12 \% \mathrm{mc})$ fumigated with sulfuryl fluoride at 0 (control), $0.5,1.0$ or $2.0 \mathrm{mg} / \mathrm{L}$ at $25{ }^{\circ} \mathrm{C}$ and $60 \% \mathrm{rh}$.

concentration, consistent with previous results with this fumigant (Hwaidi et al., 2015).

The relationship between SF concentration and both adult and egg mortality rates, $E$ and $R$, respectively, were described by first order equations (Tables 2 and 3 ) indicating the potential impact of sorption on the toxicity of SF during commodity fumigation. It has been demonstrated with several fumigants that increasing fumigant concentration (Aung et al., 2001; Baltaci et al., 2009; Daglish et al., 2002) increases mortality in the egg and other life stages. However, a decline in the efficacy of the applied concentration against eggs and adults over time due to sorption was demonstrated in this study (Tables $1-3$ ). Although it may occur, a reduction of efficacy against insects due to sorption has not been quantified for other fumigants.

Sorption caused a decline in the effective SF concentration during the fumigations, resulting in an increase in the estimated time required for control of $R$. dominica adults and eggs. For example, the time estimated for $99 \%$ control of adults fumigated with $2 \mathrm{mg} / \mathrm{L} \mathrm{SF}$ at $25^{\circ} \mathrm{C}$, was $\sim 18 \mathrm{~d}$ at filling ratio 0.5 and for eggs was $44 \mathrm{~d}$. However, under these conditions, $99 \%$ of the fumigant would be sorbed after only $34 \mathrm{~d}$ making it impossible to obtain complete kill of eggs. The results of this study indicate the effect of sorption on the exposure time, that is, a longer time of exposure combined with a higher concentration would be required for complete control of the insect eggs and adults.

The dosages of SF used in these experiments, $0.5,1$, and $2 \mathrm{mg} / \mathrm{L}$ for $168 \mathrm{~h}$, were lower than the application rates recommended for field fumigations (Agas, 2017). This was done deliberately to allow some survival of insects so that the effect of sorption of SF into grain on insect survival could be quantified. The current maximum application rate in Australia is $1500 \mathrm{mgh} / \mathrm{L}$. Although little information is available, recent research has indicated that current commercial rates are unnecessarily very high (Nayak et al., 2015). Current rates were developed for $24-48 \mathrm{~h}$ fumigations, but by increasing the exposure period, the concentration of SF can be reduced markedly below the maximum application rate. It is likely that lower rates will be adopted by industry because of the saving in costs. Thus, rates used in these experiments are relevant to future

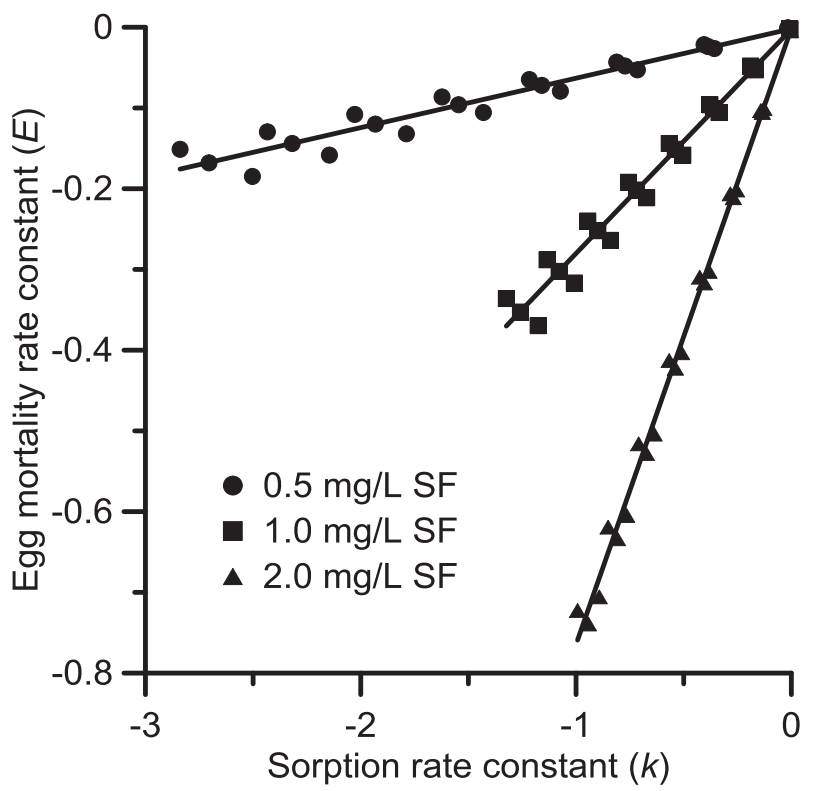

Fig. 5. Relationship between egg mortality rate constant $(E)$ for Rhyzopertha dominica, and the sorption rate constant $(k)$ of wheat $(12 \%$ m.c.) fumigated at three concentrations of sulfuryl fluoride (SF).

commercial use patterns.

Both the egg and adult mortality rates ( $E$ and $R$ ) were strongly influenced by the partition ratio of physical sorption $(K)$ (Table 3 , Fig. 3), which had an inverse quadratic relationship with $E$. An increase in $E$ was expected to occur due to the toxic effect of the gas over time. However, the continued physical sorption with time resulted in a reversal of the toxic effect and after reaching a turning point, $E$ began to decrease resulting in an increase in survival of insects when the fumigant was sorbed completely. Dumas (1980) reported that physical sorption of phosphine increased with increasing exposure time, and a similar effect was very clear in the results of the present study. Time had a strong impact on the toxicity of SF to eggs and adults, as sorption resulted in a decrease in the gas concentration to below the toxic level.

Unlike the relationship between $K$ and both $R$ and $E$, the relationship between the rate of sorption of the fumigant by the commodity $(k)$ and the mortality rate for eggs $(E)$ was linear (Fig. 5). The reaction of the fumigant with the commodity is a one directional, irreversible chemical reaction, but the rate of that reaction did not impact on $E$ under the conditions of this study. The linear relationship indicated an increase in the mortality rate, even though the reaction rate for the fumigant and the commodity increased (Table 4). It appears that although rapid physical sorption occurred in the first 24 h (Fig. 1), much of this SF was still biologically active as it had not undergone chemical reaction. The latter occurred gradually with time. These results also predict that the relationship between the sorption rate of the fumigant by the commodity $(k)$ and the mortality rate for eggs $(E)$ would become nonlinear as continuing chemical sorption with time decreases the available gas then $E$ decreases and a quadratic directional relationship with $k$ follows. That is, the chemical reaction between the gas and the commodity would continue until all the gas is sorbed completely, and the relationship would become quadratic. Further experiments are required to assess to what extent the relationship will continue to be linear, and how sorption affects the relationship between the reaction rate and the toxicity of the fumigant. Other factors that should be considered are the effects of applying higher concentrations and shorter exposure periods. The possibility of re- 
sorption of fumigant during longer exposure periods should also be investigated.

These results demonstrate that sorption is a key process that should be considered when developing fumigation protocols for SF. This is particularly important, as under-dosing due to loss of fumigant is an important factor in the development of resistance to fumigants in insect pests of stored products (Opit et al., 2012).

\section{Conclusion}

These experiments demonstrate that sorption of SF by wheat reduces the efficacy of SF against eggs and adults of $R$. dominica by removing biologically active fumigant from the system. Extending exposure time without ensuring adequate dosage or increasing concentration with an inadequate exposure time may not be sufficient to control infestations due to the phenomenon of sorption of the fumigant by the commodity. Therefore, sorption should be considered as a fundamental factor in designing SF fumigation protocols.

\section{Acknowledgements}

Mudhir Ismail Hwaidi is indebted to the Government of Iraq for providing a PhD Scholarship and to the University of New England for hosting his studies. The senior author thanks his co-authors for general supervision and the University of New England for other financial support while the senior author undertook his PhD study. We thank Hervoika Pavic and Manoj Nayak for assistance in selecting sulfuryl fluoride concentrations used in these experiments.

\section{References}

AACC, 2010. Approved Methods of the AACC. American Association of Cereal Chemists, St. Paul, MN, USA.

Agas, 2017. Approved label for sulfuryl fluoride. https://www.agasaustralia.com/ media/3707/20160303-profume-cylinder-label-australia_r2.pdf. (Accessed 12 September 2017)

Athanassiou, C.G., Phillips, T.W., Aikins, M.J., Hasan, M.M., Throne, J.E., 2012. Effectiveness of sulfuryl fluoride for control of different life stages of stored-product psocids (Psocoptera). J. Econ. Entomol. 105, 282-287.

Aung, L.A., Leesch, J.G., Jenner, J.F., Cardwell, G.E.E., 2001. Effects of carbonyl sulfide, methyl bromide and sulfuryl fluoride on fruit phytotoxicity and insect mortality. Ann. Appl. Biol. 139, 93-100.

Baltaci, D., Klementz, D., Gerowitt, B., Drinkall, M.J., Reichmuth, C., 2009. Lethal effects of sulfuryl fluoride on eggs of different ages and other stages of the warehouse moth Ephestia elutella (Hübner). J. Stored Prod. Res. 45, 19-23.

Banks, H.J., 1993. Uptake and release of fumigants by grain: sorption/desorption phenomena. In: Navarro, S., Donahaye, E. (Eds.), Physical and Chemical Processes in the Application of Controlled Atmosphere and Fumigation. Proceedings of International Conference on Controlled Atmosphere and Fumigation in Grain Storages, June 1992, Winnipeg, Canada, pp. 241-260.

Bell, C.H., Wonter-Smith, T.J., Savvidou, N., 2003. Some properties of sulfuryl fluoride in relation to its use as a fumigant in the cereals industry. In: Credland, P.F. Armitage, D.M., Bell, C.H., Cogan, P.M., Highley, E. (Eds.), Advances in Stored Product Protection, Proceedings of the 8th International Working Conference on Stored Product Protection, 22-26 July 2002, York, UK. CAB International, Oxon, UK, pp. 910-915.

Bell, C.H., Savvidou, N., 1999. The toxicity of Vikane (sulfuryl fluoride) to groups of eggs of the Mediterranean flour moths (Esphestia kuehniella). J. Stored Prod. Res. $35,233-247$.

Ciesla, Y., Ducom, P., 2009. Ministère de l'agriculture - Laboratoire National de la Protection des Végétaux - LNDS-QUALIS-71, avenue Edouard Bourlaux - BP 81-33883 Villenave d'Ornon Cedex, France, 2-1-62-5.

Collins, P.J., Falk, M.G., Nayak, M.J., Emery, R.N., Holloway, J.C., 2017. Monitoring resistance to phosphine in the lesser grain borer, Rhyzopertha dominica, in Australia: a national analysis of trends, storage types and geography in relation to resistance detections. J. Stored Prod. Res. 70, 25-36.

Collins, P.J., Daglish, G.J., Bengston, M., Lambkin, T.M., Pavic, H., 2002. Genetics of resistance to phosphine in Rhyzopertha dominica (Coleoptera: Bostrichidae). J. Econ. Entomol. 95, 862-869.

Daglish, G.J., Pavic, H., 2008. Effect of phosphine dose on sorption in wheat. Pest Manag. Sci. 64, 513-518.

Daglish, G.J., Collins, P.J., Pavic, H., Kopittke, R.A., 2002. Effects of time and concentration on mortality of phosphine-resistant Sitophilus oryzae (L) fumigated with phosphine. Pest Manag. Sci. 58, 1015-1021.

Drinkall, M.J., Pye, C.D., Bell, C.H., Braithwaite, M., Clark, S.R., Ive, J., Kershaw, S., 2004. The practical use of the fumigant sulfuryl fluoride to replace methyl bromide in UK flour mills. In: Proceedings of the 12th International ICC Cereal and Bread Congress 24-26 May, 2004, pp. 45-249.

Ducom, P., 2012. Methyl bromide alternatives. In: Navarro, S., Banks, H.J., Jayas, D.S., Bell, C.H., Noyes, R.T., Ferizli, A.G., Emecki, M., Isikber, A.A., Alagusundaram, K. (Eds.), Proceedings 9th International Conference on Controlled Atmosphere and Fumigation in Stored Products, Anatalya, Turkey, 15-19 October 2012, pp. 205-214.

Dumas, T., 1980. Phosphine sorption and desorption by stored wheat and corn. J. Agric. Food Chem. 28, 337-339.

Duong, T.M., Bui, N.T.T., Collins, P.J., 2016. Status of resistance to phosphine in insect pests of stored products in Vietnam. Indian J. Entomol. 78, 45-52. https:/ doi.org/10.5958/0974-8172.2016.00024.9 special issue (Journal of Grain Storage Research)

Edde, P.A., 2012. A review of the biology and control of Rhyzopertha dominica (F.) the lesser grain borer. J. Stored Prod. Res. 48, 1-18.

Hilton, S.J., Banks, H.J., 1997. Methyl bromide sorption and residues on sultanas and raisins. J. Stored Prod. Res. 33, 231-249.

Hwaidi, M., Collins, P.J., Sissons, M., Pavic, H., Nayak, M.K., 2015. Sorption and desorption of sulfuryl fluoride by wheat, flour and semolina. J. Stored Prod. Res. 62, 65-73.

Jagadeesan, R., Nayak, M.K., 2016. Phosphine resistance does not confer crossresistance to sulfuryl fluoride in four major stored grain insect pests. Pest Manag. Sci. 73, 1391-1401.

Kaur, R., Subbarayalu, M., Jagadeesan, R., Daglish, D.J., Nayak, M.K., Naik, H.R., Ramasamy, S., Subramanian, C., Ebert, P.R., Schlipalius, D.I., 2015. Phosphine resistance in India is characterised by a dihydrolipoamide dehydrogenase variant that is otherwise unobserved in eukaryotes. Heredity 115, 188-194.

Nayak, M., Jagadeesan, R., Kaur, R., Daglish, G.J., Reid, R., Pavic, H., Smith, L., Collins, P.J., 2015. Developing sulfuryl fluoride as a 'phosphine-resistance' breaker - the Australian experience. In: Arthur, F.H., Kengkanpanich, R., Chayaprasert, W., Suthisut, D. (Eds.), Proceedings of the 11th International Working Conference on Stored Product Protection 24-28 November 2014 Chiang Mai, Thailand, pp. 475-479.

Opit, G.P., Phillips, T.W., Aikins, M.J., Hasan, M.M., 2012. Phosphine resistance in Tribolium castaneum and Rhyzopertha dominica from stored wheat in Oklahoma. J. Econ. Entomol. 105, 1107-1114.

Osbrink, W.L.A., Scheffrahn, R.H., Su, N.Y., Rust, M.K., 1987. Laboratory comparison of sulfuryl fluoride toxicity and mean time of mortality among ten termite species (Isoptera: Hodotermitidae, Kalotermitidae, Rhinotermitidae). J. Econ. Entomol. 80, 1044-1047.

SAS Institute Inc, 1999. Version 8. Cary, NC, USA.

Su, N.Y., Scheffrhan, R.H., 1990. Efficacy of sulfuryl fluoride against four beetle pests of museums (Coleoptera: Dermestidae, Anobiidae). J. Econ. Entomol. 83, 879-882.

UNEP, 1994. Montreal Protocol on Substances that Deplete Ozone Layer: Report of the Methyl Bromide Technical Options Committee, 1995 Assessment. UNEP, ozone secrétariat, Nairobi, Kenya. http://ozone.unep.org/teap/reports/MBTOC/ MBTOC94.pdf.

Williams, L.H., Sprenkel, R.J., 1990. Ovicidal activity of sulfuryl fluoride to anobiid and lyctid beetle egg of various ages. J. Entomol. Sci. 25, 366-375. 